\title{
Design, Simulation and Validation of HMC833 Loop Filter Based on Matlab
}

\author{
Shachang Hu, Tianli Li ${ }^{*}$, Jiyue Zhang, Li Yan and Xiaojie Zhang \\ Shenzhen Key Laboratory of Electromagnetic Control, Shenzhen University, Shenzhen 518060, China
}

\begin{abstract}
In the light of the complexity of the conventional research and design of HMC833 wideband PLL, this paper studies a design method of HMC833 loop filter based on Simulink modeling in Matlab. The dynamic parameters of loop response, such as maximum overshoot, regulation time and steady-state error, are analyzed to verify the feasibility of HMC833 loop filter design.
\end{abstract}

\section{Introduction}

High temperature ceramic sensor detection system based on FPGA control needs to use FPGA as the master chip to control the high frequency signal of $5.42 \mathrm{GHz}-5.5 \mathrm{GHz}$ produced by the HMC833 chip. Then the high temperature ceramic passive wireless sensor is used for feedback collection to obtain the reflected resonant frequency, and then the real-time temperature is obtained by the relationship between resonant frequency and temperature parameters. Finally, the time curve of the resonance frequency, the real-time temperature, the S11 waveform and the temperature are displayed on the touch screen.

In order to ensure the stability of HMC833 chip to send $5.42 \mathrm{GHz}-5.5 \mathrm{GHz}$ high frequency signals, broadband PLL is used to lock frequency.

\section{Principle of HMC833}

The HMC833 chip uses a surface mount package with 40 pins, including power and ground pins, signal control pins, high frequency signal pins, low frequency signal pins, and idle pins. From the Figure 1,you can see the HMC833 internal resources and function of each module.

The external reference clock is brought into the chip by the pin XREFP.The pin CP turns the error signal of the discriminator into a current pulse, and then outputs it to an external loop filter.The filtered signal enters the on-chip VTUNE from the pin VCO. Finally, the VCO outputs a high frequency signal [1] by the pin RF-OUT through a programmable frequency division doubler.

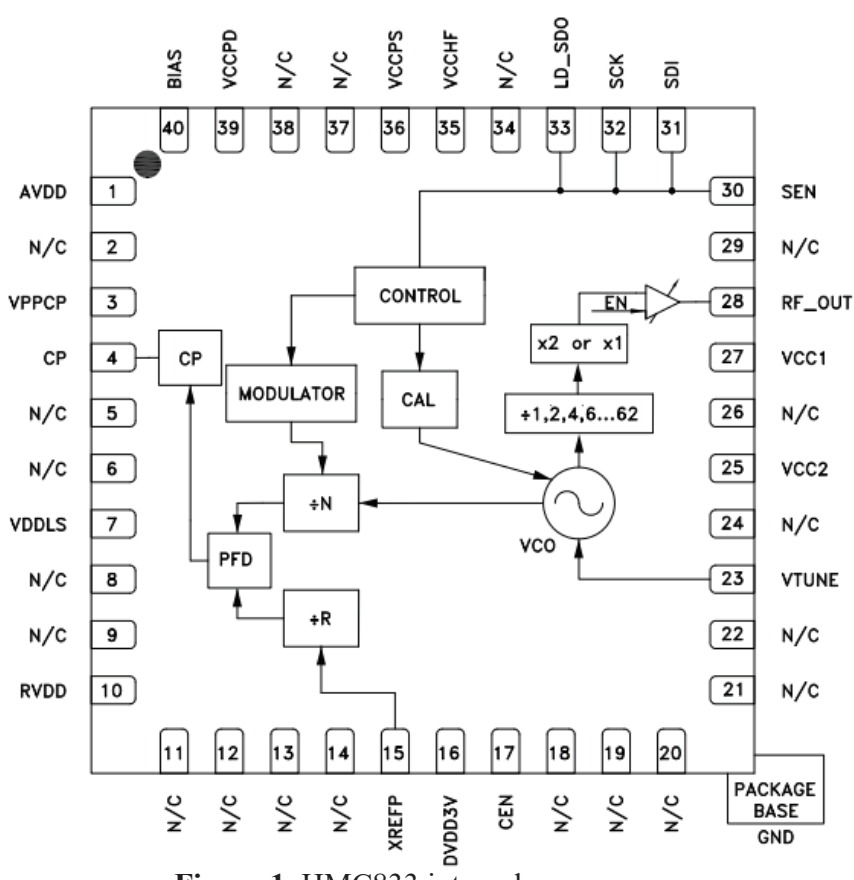

Figure 1. HMC833 internal resource

The external control signal is written to the internal registers of the chip through three pins of SEN, SDI and SCK. The pin LD-SDO can not only configure the locked output of the PLL, but also output the value of the internal register.CEN is the chip enable port, active high level. The BIAS decoupling for bias circuits requires the series connection of a capacitor to ground.

\section{Phase-locked loop}

The charge pump phase locked loop is an automatic controlled closed-loop negative feedback system. It automatically tracks the phase and frequency of the input signal. The phase of the output signal can also be locked. The principle of automatic control is its theoretical basis.

${ }^{a}$ Corresponding author: LTL988@gmail.com 
Phase frequency detector (PFD), charge pump (CP), loop filter (LPF), voltage controlled oscillator (VCO), frequency divider ( $\mathrm{N}$ frequency divider, NFD) are its basic components.It is shown in Figure 2 .

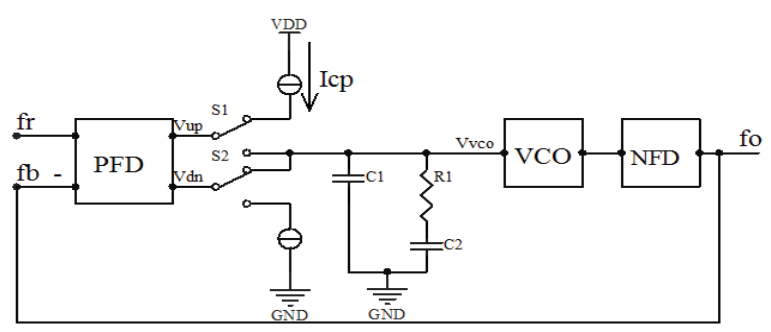

Figure 2. Schematic diagram of PLL

The complete working flow of the charge phase-locked loop is that the input $\mathrm{f}_{r}$ and the feedback voltage $\mathrm{f}_{b}$ are controlled by PFD to generate the control voltage to control the opening and closing of the S1 and $\mathrm{S} 2$ in the $\mathrm{CP}$, thus changing the charge pump current $I_{c p}$. The $I_{c p}$ is filtered by LPF to produce a control voltage $V_{v c o}$, to control the output frequency of the VCO. The frequency signal generated by VCO is divided into PFD by NFD and compared with the input $f_{r}$. So cycle until $\mathrm{f}_{r}$ equals $\mathrm{f}_{b}$.

\section{Design of loop filter}

The higher order loop filter model is evolved from the two order model. It adds two stage capacitor resistance combinations at the back of the 2 order model [2]. In the actual circuit, the first two stage filter plays a major role in the parameters of PLL, loop bandwidth, damping coefficient and phase noise. In contrast, the third and fourth stage filters in the back end are designed to optimize phase noise and improve output performance. The Simulink model in Matlab is shown in Figure 3.

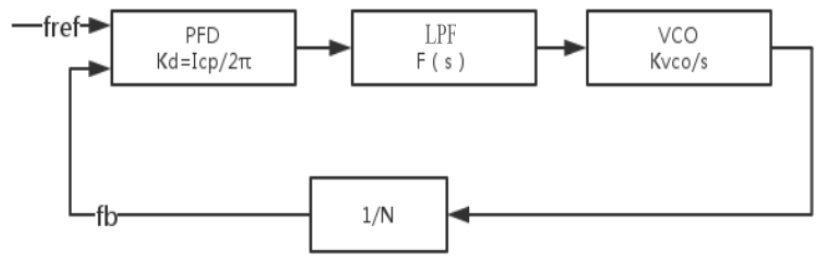

Figure 3. Simulink model

Assume the gain of the PFD is $K_{d}$, the transfer function of the LPF is $\mathrm{F}(\mathrm{s})$, the gain of the VCO is $K_{v c o}$, and $\mathrm{N}$ is the frequency division of the FD. The open-loop transfer function of the phase-locked loop can be obtained as shown in the equation [4].

$$
G(s)=K_{d} \cdot F(s) \cdot \frac{K_{v c o}}{s} \cdot \frac{1}{N}
$$

The quality of the loop filter directly affects the output characteristics of the PLL. This section is based on the maximum tuning voltage of HMC833 in the VCO chip of $5 \mathrm{~V}$, so the passive two order loop filter is adopted.

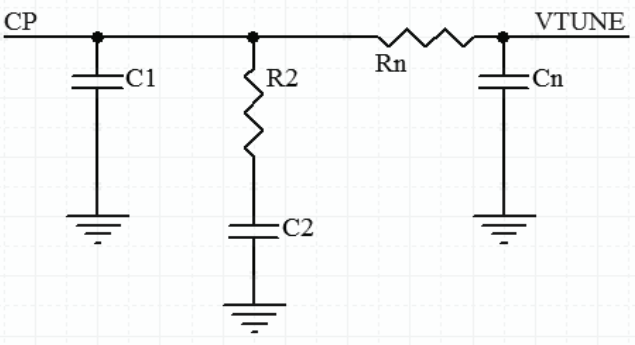

Figure 4. Passive loop filter model

According to figure 4 shows that the main elements of the second-order loop filter are $\mathrm{C} 1, \mathrm{C} 2, \mathrm{R} 2$. The ts transfer function is shown in the following formula.

$$
F(s)=\frac{1+T_{2} s}{C t \cdot s\left(1+T_{1} s\right)}
$$

$T_{1}=\mathrm{C} 1 \mathrm{C} 2 \mathrm{R} 2 / \mathrm{Ct}, T_{2}=\mathrm{C} 2 \mathrm{R} 2, \mathrm{Ct}=\mathrm{C} 1+\mathrm{C} 2 . \mathrm{The} T_{1}$ and the $T_{2}$ are loop time constant. The $\mathrm{Ct}$ is the total capacitance of the loop.

The time constant can be obtained by the phase margin equation and its partial differential equation[5]. The phase margin expression is shown below.

$$
\phi=180^{\circ}+\arctan \left(2 \pi f_{c} T_{2}\right)-\arctan \left(2 \pi f_{c} T_{1}\right)
$$

The $f_{c}$ is the Loop bandwidth.

The partial differential of the angular margin diagonal frequency:

$$
\begin{gathered}
\left.\frac{d \phi}{d w}\right|_{w=w_{c}}=\frac{w_{c} T_{2}}{1+w_{c}^{2} T_{2}^{2}}-\frac{w_{c} T_{1}}{1+w_{c}{ }^{2} T_{1}^{2}}=0 \\
T_{1}=\frac{\sec (\phi)-\tan (\phi)}{w_{c}} \\
T_{2}=\frac{1}{w_{c}^{2} \cdot T_{1}}
\end{gathered}
$$

The value of $\mathrm{Ct}$ can be approximated by the loop gain of 1 , so there are the following formulas.

$$
C t \approx \frac{K_{d} K_{v c o}}{N w_{c}^{2}} \cdot \sqrt{\frac{1+w_{c}^{2} T_{2}^{2}}{1+w_{c}^{2} T_{1}^{2}}}
$$

The $K_{d}$ is phase detector gain. The $I_{c p}$ is charge pump output current. The relation between $K_{d}$ and $I_{c p}$ is shown in the following formula.

$$
K_{d}=I_{C P} / 2 \pi
$$

The calculation of the capacitance resistance parameter can be obtained by the above method. According to the above formula, in the calculation, we need to know the gain of PLL detector, the gain and the frequency division ratio of $\mathrm{VCO}$, and the expected(1) performance parameters such as phase margin and loop bandwidth. Then the corresponding circuit element value can be estimated. If the phase margin of the phase locked loop is generally between 40 and 60 degrees, the system has better stability. The loop bandwidth is taken between one percent and $1 / 10$ of the reference frequency, and the 
phase noise of the system can be better suppressed without losing the response speed.

The actual parameters of HMC833 VCO are shown in Table 1 .

Table 1. VCO Parameters

\begin{tabular}{cc}
\hline Parameter & value \\
\hline Frequency Range & $1.5 \mathrm{GHz} \sim 3.0 \mathrm{GHz}$ \\
Reference frequency & $5 \mathrm{MHz}$ \\
Charge pump current & $2.54 \mathrm{~mA}$ \\
VCO tuning voltage & $5 \mathrm{~V}$ \\
Phase margin & $50^{\circ}$
\end{tabular}

$$
\text { Loop bandwidth }
$$

$500 \mathrm{kHz}$

Integer division ratio

53

According to table 1, the component values of the two order loop filter can be obtained, as shown in Table 2 .

Table 2. Component value

\begin{tabular}{cc}
\hline Parameter & value \\
\hline $\mathrm{C} 1 / \mathrm{pF}$ & 26.45 \\
$\mathrm{C} 2 / \mathrm{nF}$ & 1.9702 \\
$\mathrm{R} 2 / \mathrm{K} \Omega$ & 4.4389 \\
\hline
\end{tabular}

\section{Simulink modeling and simulation}

Using Matlab Simulink to build the model shown in Figure 5.

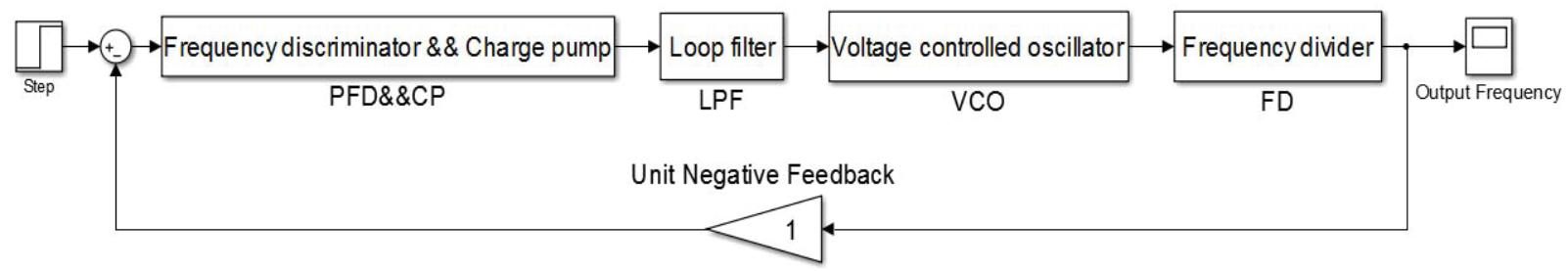

Figure 5. Simulink model.

In the Matlab simulation, the step signal is used instead of the input frequency signal fr, the frequency division ratio is 53 , and the step signal initial frequency is 4000000kHz.As shown in Figure 6, the target output frequency is $5512028 \mathrm{kHz}$. For ease of observation, the image is magnified at the time axis 20us. In Figure 6, ordinates represent frequency values, and abscissa indicates time.

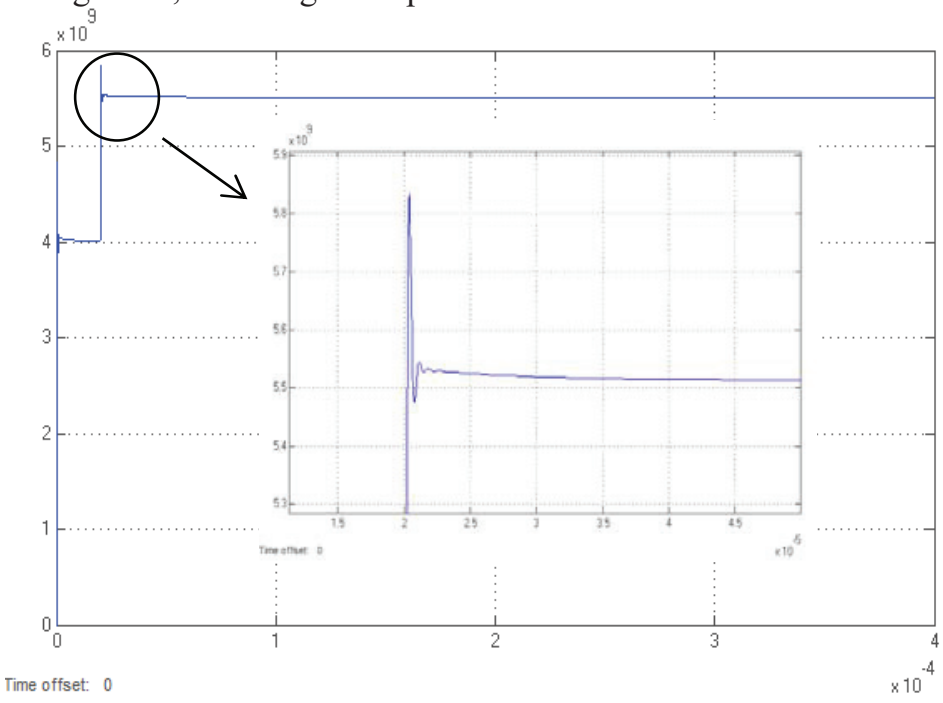

Figure 6. Time domain response

For accurate index analysis, the input signal is set to the unit step signal in Matlab simulation. The time domain step response curve and the open-loop response in the frequency domain are shown in Figure 7, Figure 8.

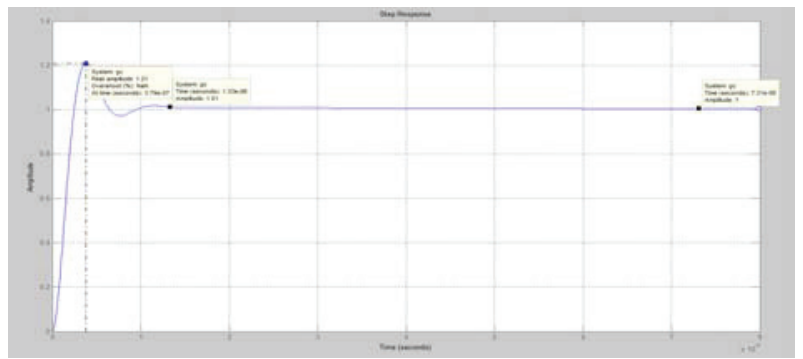

Figure 7. Unit step response 


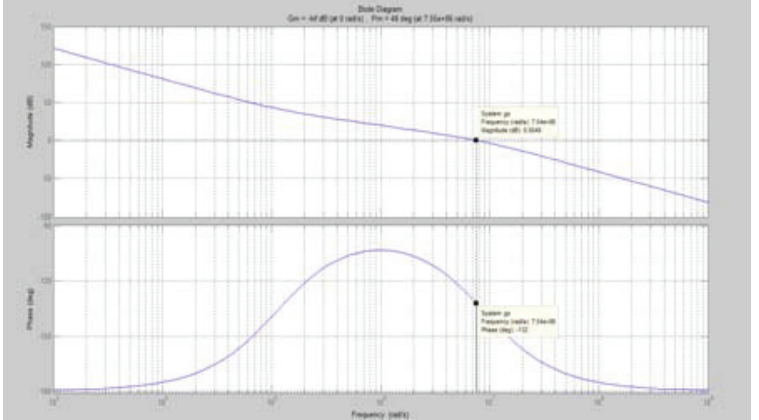

Figure 8. Open-loop response

\section{Simulation results analysis}

Figure 6 shows the two oscillations, the first oscillation that frequency from $0 \mathrm{~Hz}$ jump to the starting frequency of $4000000 \mathrm{kHz}$ process. And the second oscillation that frequency from $4000 \mathrm{MHz}$ jump to the target output frequency $5512028 \mathrm{kHz}$ process. Figure shows that the system maximum overshoot is $5.82 \%$, the system reach the steady-state value of $5512028 \mathrm{kHz}$ at $0.35 \mathrm{us}$.

When the unit step signal is used as input, the responses obtained are shown in Table 3.

Table 3. Response indicator

\begin{tabular}{cc}
\multicolumn{2}{c}{ Table 3. Response indicator } \\
\hline Index & Value \\
\hline Steady state error & 0 \\
Maximum overshoot & $20.96 \%$ \\
Rise time/us & 0.247 \\
Adjustment time/us & 0.845 \\
Peak time/us & 0.382 \\
PM Frequency/(rad/s) & $7.5463 \mathrm{e}+06$ \\
Phase Margin $/\left(^{\circ}\right)$ & 47.9678 \\
Delay Margin $/(\mathrm{rad} / \mathrm{s})$ & $1.1094 \mathrm{e}-07$ \\
Stable & 1 \\
\hline
\end{tabular}

From the above results, it can be analyzed that the maximum overshoot of the system is $20.96 \%$, the regulation time is $0.845 \mathrm{us}$, and the steady-state error is zero. This system has faster response speed, higher precision and meet the performance requirements of PLL frequency. Thus, it is proved that the design method of HMC833 loop filter is feasible.

\section{Experimental verification}

The experimental platform and the real-time output frequency display are shown in Figure 9.

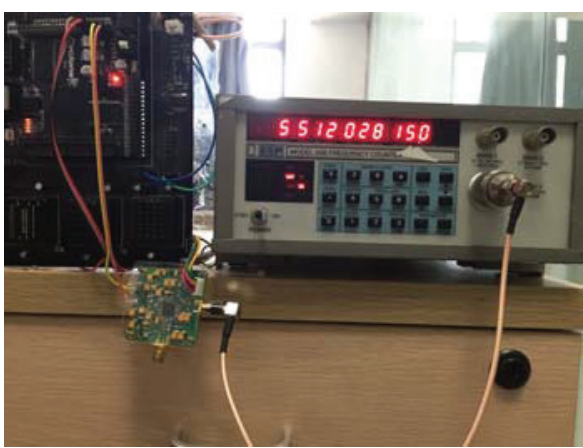

Figure 9. Real-Time output frequency

The HMC833 is controlled by FPGA with an output frequency of $551208 \mathrm{Khz}$. The output frequency error curve that runs for 10 minutes is shown in Figure 10.

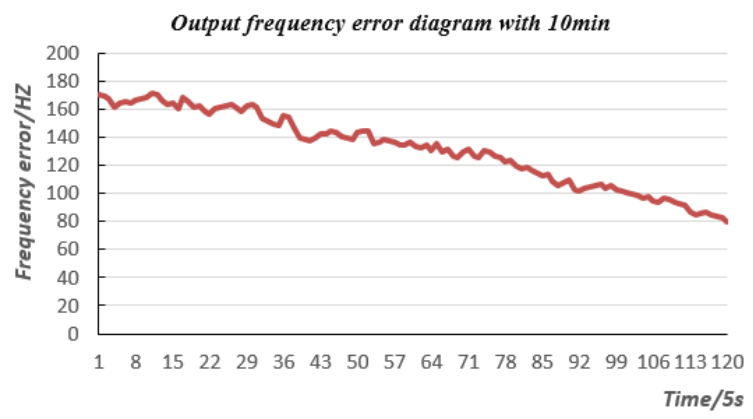

Figure 10. Output frequency error

The output frequency jump margin is $12 \mathrm{~Hz}$ and the frequency lock accuracy is $1 \mathrm{kHz}$. It has the characteristics of good stability and high steady-state precision. It satisfies the performance requirements of CPPLL for locking frequency, which verifies feasibility of the design method of HMC833 loop filter.

\section{References}

1. J.K. Tian, J.K. Yan, T. Chu, H.Z. Chen. Programming and analysis of wideband frequency synthesizer, Electronic Measurement Technology (2014).

2. F.M. Gardner. Charge-Pump Phase-Lock Loops, IEEE Trans. Commun, 28.11(1980):1849-1858.

3. J.J. Yao, et al. Design and analysis of VCO in PLL Circuit, Electron. Des. Eng (2011).

4. S. Mirabbasi, K. Martin. Design of loop filter in phase-locked loops, Electron. Lett, 35.21(2002): 1801-1802.

5. J.B. Lan, et al. A nonlinear phase frequency detector for fast-lock phase-locked loops, IEEE, International Conference on Asic IEEE, 2009:1117-1120. 